\title{
Towards a System of Guidance, Assistance and Learning Analytics Based on Multi Agent System Applied on Serious Games
}

\author{
Lotfi Elaachak, Amine Belahbibe, Mohammed Bouhorma \\ Computer science, systems and telecommunication Laboratory (LiST) \\ Faculty of Sciences and Technologies, Abdelmalek Essaadi University \\ Tangier, Morocco
}

\section{Article Info \\ Article history: \\ Received Jan 6, 2015 \\ Revised Feb 12, 2015 \\ Accepted Feb 26, 2015}

\section{Keyword:}

Educational data mining

E-learning

Learning analytics

Multi agent system

Rule based inference engine

Serious games

\begin{abstract}
With the revolution that the education field has known concerning the new tools and manners of learning and especially the integration of new technology as tools of teaching, several new tools have appeared and among these tools there are serious games, serious games as new tool dedicated to education have occupied an important place, and replaced other tools often used in the learning process. But in the order that serious games reach the intended objectives and help instructors to achieve their perspectives considered, it must be that this kind of video games will be equipped with a guidance and assistance system that will assist the learners during the progression of a sequence of the video game, and in addition they will be equipped with a system of learning analytics that will help instructors to improve the learning process and teaching methods according to the performances and feedbacks of their learners. In this perspective of research and development we will establish in this paper a new system of assistance, guidance and learning analytics based on a multi agent system that will work in tandem with a serious game.
\end{abstract}

Copyright () 2015 Institute of Advanced Engineering and Science. All rights reserved.

\section{Corresponding Author:}

Lotfi Elaachak,

Computer science, systems and telecommunication Laboratory (LiST),

Faculty of Sciences and Technologies, Abdelmalek Essaadi University

Tangier, Morocco.

Email: lotfi1002@gmail.com

\section{INTRODUCTION}

Serious games or learning video games have for mission the transfer of the knowledge in a fun and interactive way; this feature has made this kind of video games one of the most powerful tools used for teaching and learning, and it is within the scope of using the new technologies in the educational field. For serious games prove their effectiveness; they must be able to transmit knowledge properly and allow measuring and analyzing the learning outcomes. Unfortunately, it is a challenge in any video game to teach a player how to play and to guide them through the game world., especially when dealing with a serious game, the challenge intensifies due to the inherent variations in student backgrounds, making the choice of how to guide the student from the start to the end of the game without direct instructor interactions a complex problem [1]. In addition, the difficulties in measuring learning outcomes achieved through serious games use have been a main barrier for successful deployment and adoption of such video games within formal education [2]. There are several research works was directed by different institutions and laboratories, concerning the inclusion of assistance and learning analytics system in serious games, among the realization that exist there is a virtual instructor enabled mobile augmented reality learning "MARL" games have the potential to provide a fun and educational experience. This kind of video games assist learning conceptual 
knowledge as well as psychomotor task in real world environments [3]. In the literature there are also methods and studies on the interaction of serious game with learning analytics system [4-7].

With the aim to overcome the difficulties cited above, therefore, we will detail in this paper the different steps of the establishment of a system that will be able to react with the learners by giving them information or assistance during their progression in the video game, this relationship will help learners to improve their learning level and also their performance. On the other hand, we will detail also the establishment of the system capable of saving data about learners in order to analyze them by using several algorithms, specific methods and techniques, the results of such analyzes will help the instructors to improve both their strategy and their teaching methods, based on the feedback of their learners, the inclusion of such system will make serious games a complete tool suitable for the process of learning. In this perspective of research and development we aim in this paper to develop a serious games about a waste sorting process, this video game will be equipped with a multi agent system composed of several intelligent agents, which each agent in the system has a specific task, there is an agent that assists players according to their performances during a sequence of video games, there is also an agent that extracts and analyses data concerning the learners in order to be interpreted by the instructors.

\section{THEORETICAL BACKGROUND}

The establishment of such system requires a specific architecture with the interaction of several technologies; therefore, we have used the combination of the rule-based inference engine, the educational data mining tool with the learning analytics techniques, all of those technologies will be hosted in different agents of the multi agent system, in this section we will detail the theoretical background of the multi agent system architecture and the technologies used in the establishment of the proposed system.

\subsection{Rule-Based Inference Engine}

The main role of the inference engine is derived new facts from the knowledge base as shown in the Figure 1, otherwise the inference engine applied logical rules to the knowledge base, then inferred new knowledge, there are different types of inference engines, but in this paper we will focus on rule-based inference engine who applies several rules with data to derive new facts. This kind of the inference engine is always composed of three components, the interpreter that executes the chosen agenda elements by applying the corresponding base rules, the scheduler that maintains the control over the agenda by estimating the effects of applying inference rules in criteria on the agenda, the consistency enforcer that attempts to maintain a consistent representation of the emerging solution.

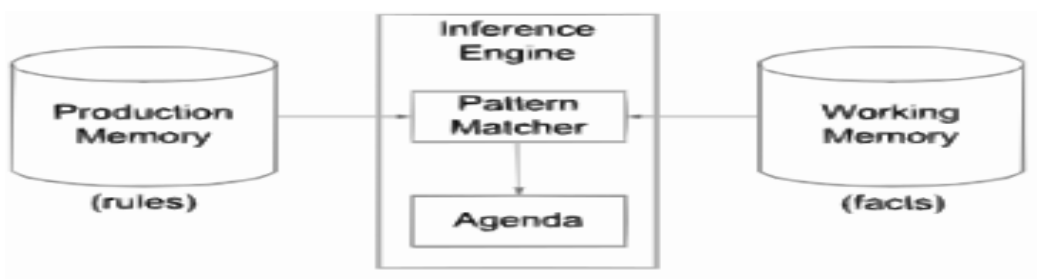

Figure 1. Inference engine architecture

Generally, in every rule-based inference engines, there are two kinds of inference, backward chaining and forward chaining. The forward chaining, according to this algorithm the inference is inference is triggered by the arrival of new data in working memory; it's also called data-directed inference. The backward chaining where inferences are not performed by the system is made to prove a particular goal; it's also called hypothesis driven, or goal directed inference.

\subsection{Educational Data Mining}

The Data Mining is the process of analyzing data from different perspectives and summarizing the results as valuable information. It has been defined as the nontrivial process of identifying valid, potentially useful, novel and ultimately understandable patterns in data $[8,9]$. Among the most famous branches of data mining there are the educational data mining "EDM" that describes a research field concerned with the application of data mining, machine learning algorithms and statistics tools to information generated from educational area. Other definition of educational data mining as tool of Mining in educational environment, concern with developing new methods to discover knowledge from educational databases [10]. It's an

Towards a System of Guidance, Assistance and Learning Analytics Based on Multi Agent ... (Lotfi Elaachak) 
emerging discipline, concerned with developing methods for exploring the unique types of data that come from educational parameters, and using those methods and techniques to better understand students, and the settings which they learn in [11]. There are several data mining techniques used to extract useful data that help users to predict unknown or future values of the attributes, and also to describe the data in a manner understandable and interpretable to users. Among those techniques there is Classification, Clustering, Association rule, Regression, and others machine learning algorithms like neural networks, Bayes network, etc.

\subsection{Learning Analytics}

The learning analytics research trays to answer increasingly several questions about what a learner knows and whether a learner is engaged. The application fields of the learning analytics concern modeling of user knowledge, user behavior, and user experience, user profiling; modeling of key concepts in a domain and modeling a domain's knowledge components, and trend analysis. Below we will detail those concepts.

The user knowledge modeling is the Collection of user's skills and knowledge extracted from different data sources, looking at the registered data that represents the interaction between user and both learning system or serious games. Among the information extracted there are: correctness of a student response alone or in a series, time spent on practice before attempting to answer question or to do manipulations, number and nature of hints requested, repartition of wrong answers and errors made. Such inferences can be model by a predictive model or by teacher looking at student data on dashboard. A popular method for estimating students' knowledge is Corbett and Anderson's [12] knowledge tracing model, an approach that uses a Bayesian-network-based model for estimating the probability that a student knows a skill based on observations of him or her attempting to perform the skill. More recently, a new study [13] has proposed a new method for knowledge tracing using a machine learning approach to make contextual estimations of the probability that a student has guessed or slipped. Incorporating models of guessing and slipping into predictions of student future performance was shown to increase the accuracy of the predictions by up to 48 percent. The User behavior modeling in education often characterizes student actions as on- or off-task and can be used as a proxy for student engagement. It relies on the same kinds of learning data used in predicting user knowledge plus other measures, such as how much time a student has spent online, whether a student has completed a course, documented changes in the classroom or school context, attendance, tardiness, and sometimes a student's level of knowledge as inferred from his or her work with the learning system or from other such data sources as standardized test scores. Baker and colleagues have conducted a series of studies on detecting and adapting to students' off-task behaviors called gaming the system in adaptive learning systems that teach algebra [14].The User experience modeling ascertaining whether a student is satisfied with the learning experience can be judged by students' responses to follow-up surveys or questionnaires and by their choices, behaviors, performance, and retention in subsequent learning units or courses. User experience through methods other than data mining, collected time spent on redesigned course components, periodic surveys of students' motivation state during the course, and learning performance. The user profile is a collection of personal data describing the essential characteristics of a user. User profiling refers to the process of constructing and applying student or group profiles using data mining and machine learning algorithms. In educational data mining techniques, such as classification and clustering, are often used to categorize learners based on the kinds of personal learning data.

\subsection{Multi Agent System}

A multi agent system "MAS" is a computing distributed system, composed of a number of interacting computational entities, but the single difference between classical distributed systems and multi agent system is that the entities that interact in the system, are intelligent. These entities that react in the system are called agents, and must be able to communicate each other. The concept of multi agent system has influenced the initial developments in areas like cognitive modeling and instructional design $[15,16]$. Until nowadays, the multi agent systems establish a major research subject in distributed artificial intelligence. MAS technology aims to create general and specialized behavioral and interaction models and to implement these models into distributed and interacting computer programs called agents. The design of such models follows certain guidelines that characterize agents [17]. The MAS have been used in many fields to simulate complex systems, such as decision support tools for distributed decision problems.

\section{THE SERIOUS GAME}

Waste sorting has and should become part of our daily life to improve our living environment. With the importance of the waste sorting and the benefits that it presents, to this effect many instructional experts have found that teaching the basics of waste sorting for kids dice their young age can be beneficial for the 
environment and the economy. For this reason there is no more robust way to learn the basics of waste sorting better than the video games, view to their advantages such interactivity and playability that attracts the intention and the desire into the players to play more. In this perspective of development our research team has developed an interactive web based serious game for waste sorting dedicated for children that will be described in this section.

\subsection{The Concept of Waste Sorting Serious Game}

The main objective of the proposed serious game Figure 2 is to teach kids about recycling different waste. The player should sort different waste into trash, paper, plastic, metal, glass, and organic, etc. The sorting is done by catching different objects generated randomly and dropping in the appropriate container according to their types, this mechanism will be done by using a tool called a leap motion controller. The waste sorting serious game will be equipped by the timer, and the assessment system that evaluates the players according to their performances if they make a good choice the reward will be the gain of some points, although if the opposite case the punishment will be the loss of some points. With the assessment system, the timer, and the interactivity based on hand movement the proposed serious game will be more challenging and attractive especially for kids, it will allow them to live a beneficial and unforgettable experience. The proposed serious game has been developed by JavaScript API, therefore, it need just a web browser to be run.

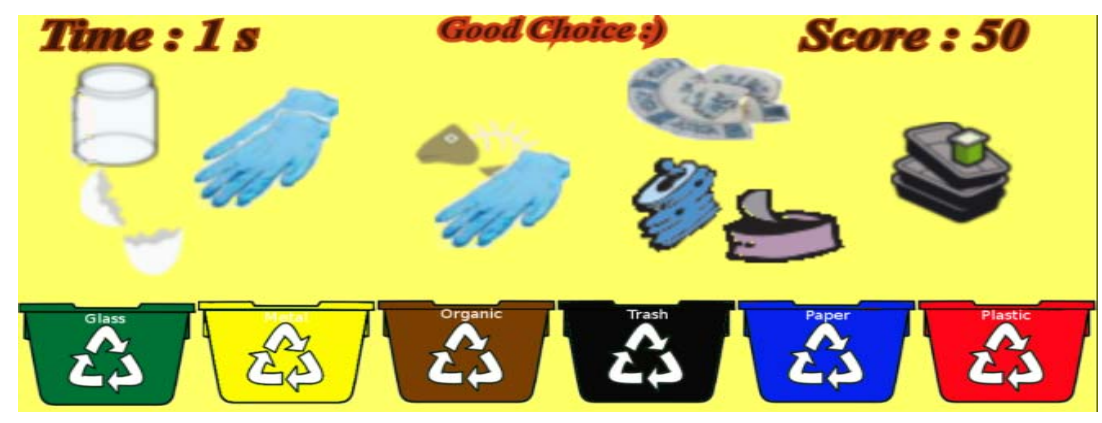

Figure 2. Screen shoots from waste sorting video game.

\subsection{Interactivity with Leap Motion Controller}

The Leap Motion controller is a small device that can be connected to a computer using a USB. It uses infrared (IR) imaging to determine the position of predefined objects in a limited space in real time. It can then sense hand and finger movements in the air above it, and these movements are recognized and translated into actions for the computer to perform. According to the official information founded in the official web site of leap motion [18], the Leap software analyzes the objects observed in the device's field of view. It recognizes hands, fingers, and tools, reporting discrete positions, gestures, and motion. The controller's field of view is an inverted pyramid centered on the device Figure 3 . The effective range of the controller extends from approximately 25 to 600 millimeters above the device. The controller itself is accessed and programmed through Application Programming Interfaces (APIs), with support for a variety of programming languages, ranging from $\mathrm{C}++$ to Python and JavaScript. The positions of the recognized objects are acquired through these APIs. The Cartesian and spherical coordinate systems used to describe positions in the controller's sensory space.

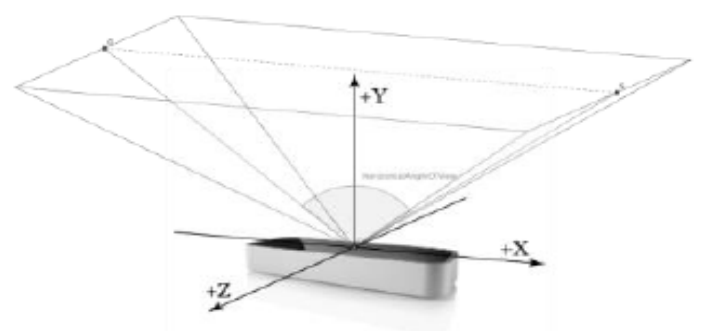

Figure 3. Leap motion's field of view

Towards a System of Guidance, Assistance and Learning Analytics Based on Multi Agent ...(Lotfi Elaachak) 
With the features that leap motion controller offers and with the use JavaScript application programming interface, we have integrated it in the proposed video game, therefore, the player has to move his hand and catch the random generated objects in order to drag and drop them, then place them in a correct container. With this possibility the proposed video game will become more interactive and so close to the real case. That will create envy into the player to play more. In addition, this concept will allow us to save all gestures done by the players during a sequence of video game; this data will be used by educational data maiming to understand the player's behaviors and also to analyze their performances.

\section{THE ESTABLISHMENT OF THE SYSTEM}

The system of guidance, assistance and learning analytics proposed in this paper will be established via the use of the multi agent system; the chosen system is composed of several entities called intelligent agents, where each agent of the system is equipped with a rules engine, data mining algorithms or the methods of the learning analytics. As show in the Figure 4 there are different technologies used in the architecture of the proposed system, in the first place we have a web application deployed in application server, this web application is composed of a web based serious game that interact with the server side by using Ajax technology, the use of the interactive interface allows real time interaction between the actions and the gestures of the learners and the appearance of the assistance messages that the system should provide to the learners to guide them in order, to improve their performances.

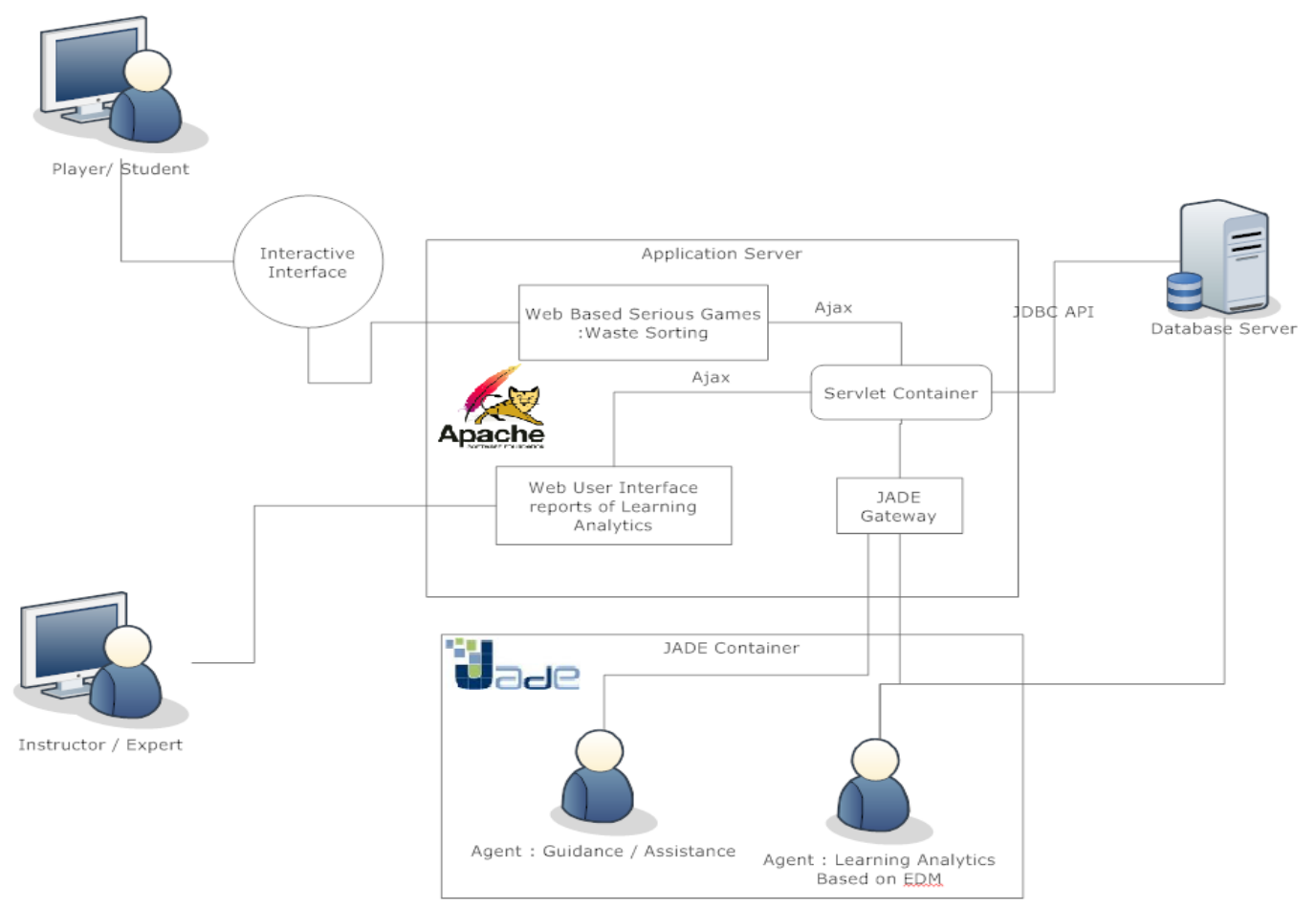

Figure 4. Architecture of the system based on MAS for guidance and Evaluation Concerning Serious Games Players.

Regarding the multi agent system part, Jade [19] has allowed us an easy implementation of the multi agent system "MAS", as presented in the schema our MAS is composed of tow intelligent agents; the first one is the guidance and assistance agent; by against the second agent is for learning analytics. Each agent reacts in its own way and implements its own algorithms to ensure the proper functioning of the whole system. As mentioned before the first agent is equipped with a rules engine, in order to build such engine we have chosen drools [20] a Business Rules Management System "BRMS" solution. It provides a core Business Rules Engine"BRE". We have established a rule tree Figure 5 that contains all the possible cases of figures that will guide and assist learners during their video game sequence and according to their performances. In the rules tree there are several elements, in the level one that represents the time of the serious game 
sequence, as presented in the tree there are three intervals of the time $[120 \mathrm{~s}$ to $90 \mathrm{~s}]$, [90s to $30 \mathrm{~s}]$ and [30s to $0 \mathrm{~s}]$. In the level tow represents the Number of hand gestures " $\mathrm{NG}$ " done by the player to place different objects in the several containers. In the level three "\%NF" represents the number of faults divided by the number of gestures. In the level five "CF" represents the container that has the largest number of faults committed by learners. As last elements there are several messages that will be displayed on the screen to assist the learners. All the messages shown by the rules engine are detailed in the table 1. The agent intercepts all the actions and gestures of the learners, and then interprets all the data to give the correct message that will be displayed on the screen, and then the learner will react according to the message already displayed on the screen, with the aim to improve his learning process.

Table 1. Messages shown by the system to guide learners during their sequence of serious game

\begin{tabular}{|c|c|}
\hline $\mathrm{N}^{\circ}$ & Message \\
\hline MSG1 & You need to focus more to have a good result, you are in the beginning you can do better. \\
\hline MSG2 & Good performance up to now, try to keep the same level of concentration to get the good result. \\
\hline MSG3 & You have to focus more, your result is too bad, and your choices are random. \\
\hline MSG5 & You have to do more effort, and move your hands to collect more objects. \\
\hline MSG6 & The Glass waste is composed of bottles, cups, etc., you have to put those objects in the Green container. \\
\hline MSG7 & The Metal waste is composed of cans, metallic objects, you have to put those objects in the yellow container. \\
\hline MSG9 & $\begin{array}{c}\text { The Trash waste is composed of anything not recyclable like tires, plastic bag, etc. You have to put those objects in the } \\
\text { black container. }\end{array}$ \\
\hline MSG10 & The Paper waste is composed of books, news paper, etc. You have to put those objects in the blue container. \\
\hline MSG11 & The Plastic waste is composed of different of plastic objects; you have to put those objects in the red. \\
\hline MSG12 & $\begin{array}{c}\text { You have done a lot of gestures and your percentage of good choices is low, try to focus more during the choice of } \\
\text { objects. }\end{array}$ \\
\hline
\end{tabular}

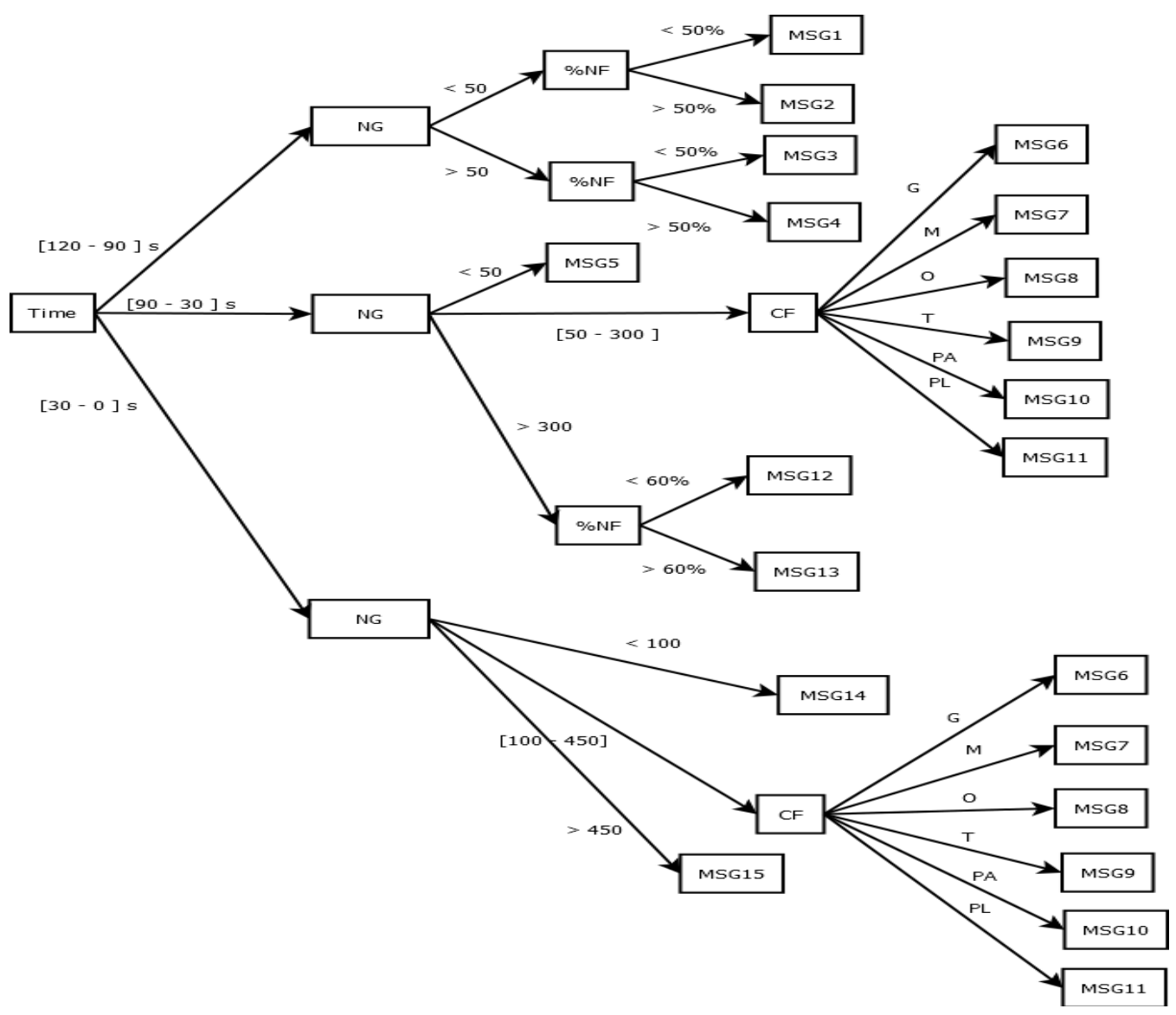

Figure 5. Scheme represents a rule tree that will guide learners. 
The second agent will feed a backend web-based application; this application will be used by the teachers and trainees. Its main role is to give a global view concerning the learners that play the video game, and show graphs and dashboard of learning analytics. The scheme in the Figure 6 represent the data base that will save all the data concerning learners, this data will be interpreted by the educational data mining algorithms, we have used clustering algorithm e.g. "K-means algorithm" that will cluster the learners according to their performances and the result will be presented in pie flowchart. In addition the data recorded in the data base will be interpreted by learning analytics algorithms based on user profiling method and sort players since their score, number of gestures, etc. there is other information will be given by the agent and learning analytics methods like grouping different kind of containers according to the number of good and bad choices done by the learner. All of this information will be viewed by the teachers in order to make a good decision to improve their manner of teaching.

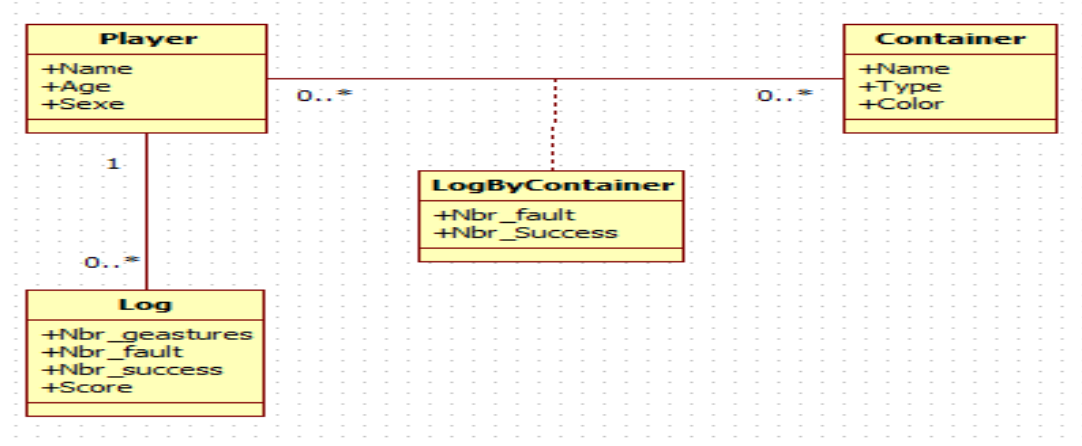

Figure 6. The scheme of the data base

The communication between the web applications that hosts the web-based serious game and different agents are provided by the use of the jade gateway. This layer will ensure the communication between those separated systems and will allow them to function in harmony. In the next section we will detail the dashboard of the learning analytics, and also the kind of messages given by the rule engine, that will guide and assist the learners.

\section{RESULTS}

The intended result is a system composed of a web-based application with a multi agent system, the web-based application deployed in the server application was developed by using several technologies and tools e.g. "JavaScript APIs, Ajax, Java Technologies, Jade, Drools, and Weka [21]". The combinations of these technologies have ensured the proper functioning of the system and despite complexity associated with the implementation of such a system. The concerned web-based application is composed of two parts; the first part is a web-based serious game developed by using several JavaScript APIs that allow the creation of $2 \mathrm{~d}$ video games on the web; therefore, it needs just a web browser to be run, in addition, the interaction between learners and serious game is done through an interactive interface "leap motion controller" based on the hands and finger gestures. The Figure 7 shows a screenshot from the proposed web-based serious game about the waste sorting process; with the assistance's messages displayed on the screen to guide learners according to their performances and their progression in the video game. The second part is a backend webbased application is a dashboard used by teachers and trainees in order to have a global view on their students; they can measure and analyze the performance of their students by using a combination of several educational data mining algorithms and learning analytics methods. 


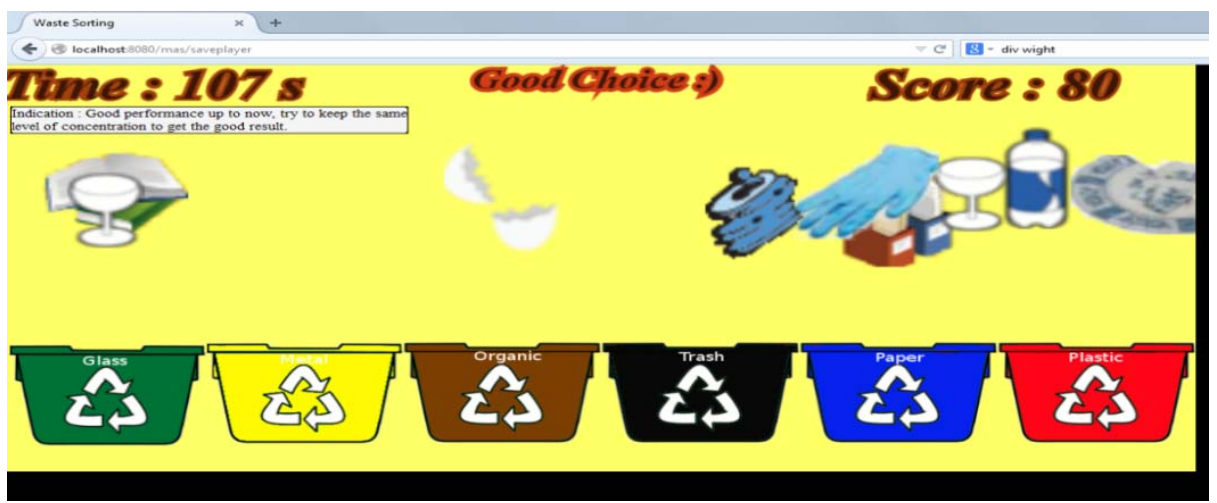

Figure 7. Screenshot of the web-based serious game with apparition of assistance message

The Figure 8 shows a pie chart about the user profiling that clusters several groups of learners according to their performances. The pie chart was fed by k-means cluster algorithm, in this pie chart there are four groups of the learners grouped according to theire performances, the orange part of the pie chart represents the good learners who understood all the basics of waste sorting, view to their score and the number of their hand gestures. For the learners that belong to the black part of the pie chart, are generally good, but they need some guidance and explanation about the basics of the waste sorting. Taking the case of the green part of the pie chart the learners that belong to this part, have an average level, they have few problems regarding their understanding about the proposed topic and they need an explanation and assistance to understand the basics of waste sorting. For the last part the blue part the most of the learners that belong to this part of the pie chart have several problems and difficulties, they have chosen the objects randomly and without thinking. They need explication on basics of waste sorting, and a special assistance, in order, to increase their level of comprehension. In the same web interface there is a graph about a number of good and bad choices grouped by the type of the container. There is also a web interface that shows a graph about the learners' information in this case we have presented the number of gestures and total score of each learner, as shown in the Figure 9. This representation gives a global view on the activity of each learner that plays the serious game, and with this dashboard the instructor can make decision, in order to improve and change his manner and strategy of teaching with the aim of transmit knowledge in a correct way.

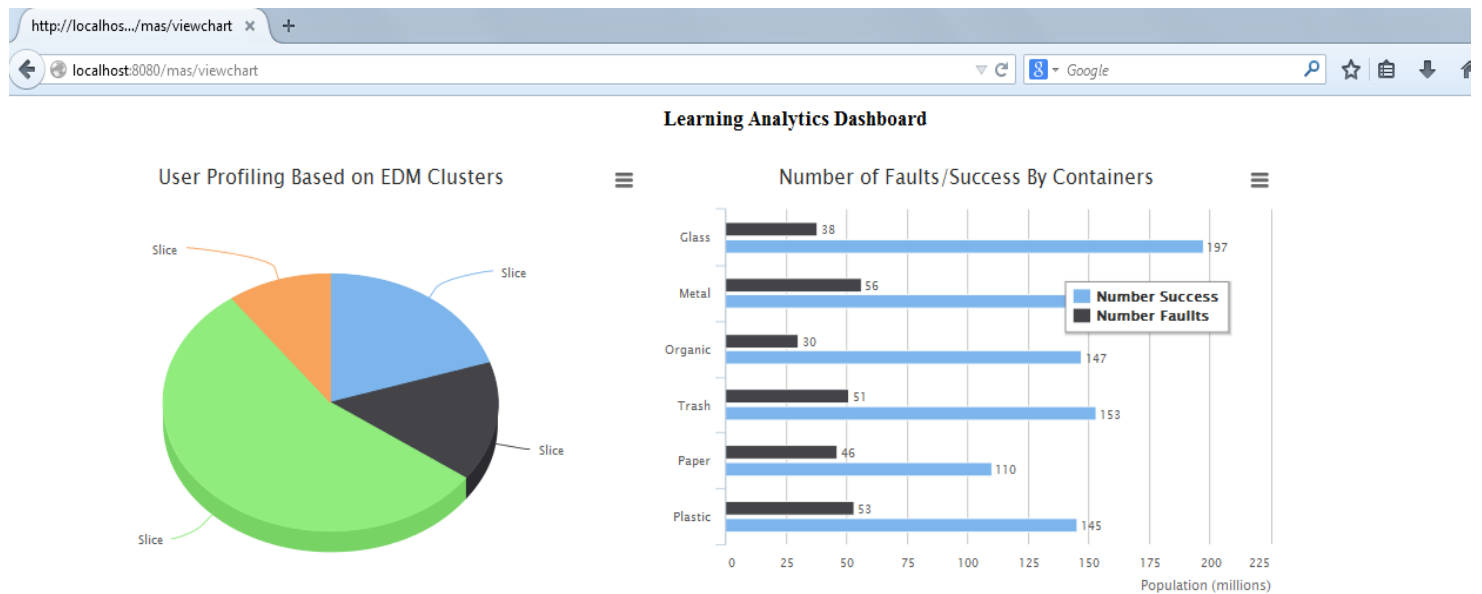

Figure 8. Screenshot of the dashboard concerning User profiling and Number of faults and success by containers 


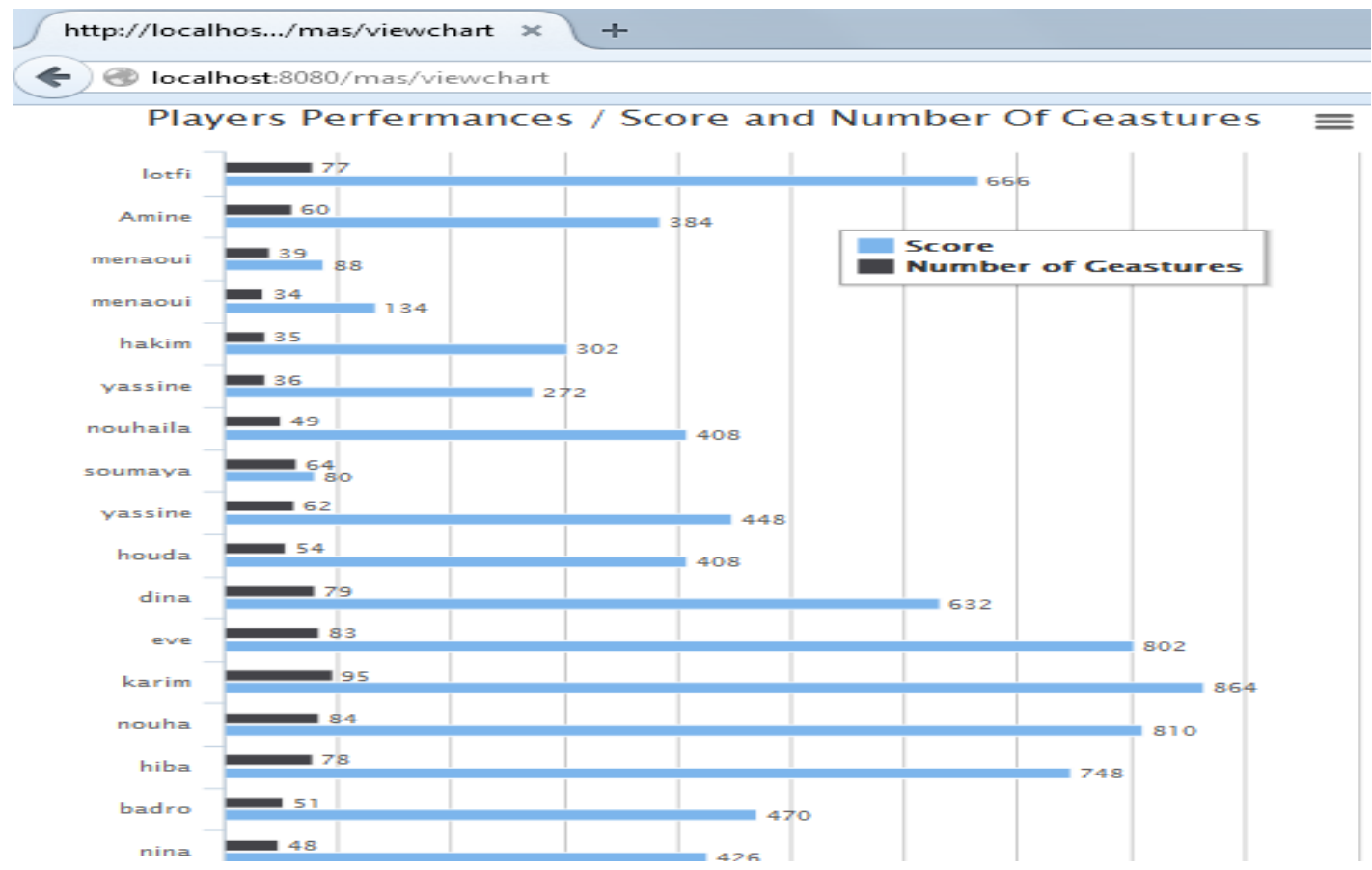

Figure 9. Screenshot of the dashboard concerning learners's performances

The serious game and the backend web-based application communicate with guidance/ assistance agent and learning analytics agent via a Jade Gateway, this interface insures a real-time communication between two heterogeneous environments web and multi agent system. with this complementary system that will enable learners to increase their level of learning and their level of understanding based on the messages and guidance offered by the system during a sequence of video games, although the application Dashboard will enable teachers to adapt their teaching strategies and methods based on performance and the results of their students.

Comparing the approach followed in this paper to establish the leaning analytics system, with other approaches in other contributions [22,23], we can conclude that the main objective of the proposed work and other contribution is to simplify teachers' task when using serious games by providing real-time information of the actual learner' use of the games while in the classroom. But the concept and the technologies used are different, in the other contribution the authors have used XML technology to deliver assessment data to the teachers, by cons in the proposed paper we have used a combination of educational data mining and learning analytics to extract benefit information to the teachers in order, they have a global view about the level of their learners, and will allow them to properly identify learners gaps. Regarding the assistance and guidance system the study about solaris one serious game [1] the the authors seek to first assess the student's skill to ascertain the amount and type of help to provide. Before each game, a set of multiple-choice questions are presented to find out what the student knows and what they may need help. By cons our system represents an adeventage because it reacts with learners automatically and according to their progress in the video game, which will allow learners to live a beneficial experience.

\section{CONCLUSION}

In this paper we have detailed the establishment of guidance, assistance and learning analytics system based on a multi agent system, it will work in collaboration with a serious game developed by our research team. The proposed system has several advantages and benefits that concern both learners and instructors. One of the most important advantages of our system is it efficiency. In one hand it can assist and guides the learners, which will improve their level of understanding and accelerates their learning process and their analytical skills, our system provides an environment both interactive and entertaining that carefully target the knowledge that the learner must acquire. In the other hand it will allow teachers and instructors to make their decisions easily, in order to improve their teaching methodologies, strategies and teaching manners, the decision will be made with the help of the dashboard that has several graphs and flowcharts 
which are fed by the learners' data interpreted via both educational data mining algorithms and leaning analytics methods. Among the perspectives envisaged there is the evolution of the current system by adding other agents equipped with other algorithms of the educational data mining, other learning analytics methods, and machine learning algorithms, the implementation of such system will open a new trend towards smart serious games.

\section{ACKNOWLEDGEMENTS}

This research paper is made possible through the help and support from the students of computer engineering. We gratefully acknowledge the support of the bachelor, master students and all other participants.

\section{REFERENCES}

[1] Yang T, Kauser J, Sachin S, Christopher JF. Solaris One - A Serious Game for Thermodynamics. 121st ASEE Annual conference \& Exposition. Indianapolis. 2014.

[2] J. Baalsrud Hauge, F. Bellotti, R. Nadolski, M. Kickmeier-Rust, R. Berta, M. B. Carvalho. Deploying Serious Games for Management in Higher Education: lessons learned and good practices. Proceedings of the 7th European conference on games based learning, 2013, pp. 225-234.

[3] Jayfus D, Kathleen H. Extending the 'Serious Game' Boundary: Virtual Instructors in Mobile Mixed Reality Learning Games. Digital Games Research Association Conference. 2007.

[4] Reese, D. (2014). Digital Knowledge Maps: The Foundation for Learning Analytics Through Instructional Games. In D. Ifenthaler \& R. Hanewald (Eds.), Digital Knowledge Maps in Education (pp. 299-327): Springer New York.

[5] Minović, M., Milovanović, M. Real-time learning analytics in educational games. Paper presented at the 1st International Conference on Technological Ecosystem for Enhancing Multiculturality. (2013).

[6] Fulantelli, G., Taibi, D., \& Arrigo, M. A semantic approach to mobile learning analytics. Paper presented at the 1st International Conference on Technological Ecosystem for Enhancing Multiculturality. (2013).

[7] Greller, W., Ebner, M., \& Schön, M. Learning Analytics: From Theory to Practice-Data Support for Learning and Teaching Computer Assisted Assessment. Research into E-Assessment (pp. 79-87): Springer. (2014).

[8] Fayyad, U. M., Pitatesky-Shapiro, G., Smyth, P., and Uthurasamy, R. (1996). Advances in Knowledge Discovery and Data Mining, AAAI/MIT Press.

[9] Frawley W., Piatetski-Shapiro G. and Matheus C. (1992). Knowledge discovery in databases: an overview. AI Magazine. 14(3). 57-70.

[10] Erdogan \& Timor. (2005). A Data Mining Application in A Student Database. Journal of Aeronautic and Space Technologies. July 2005 Vol. 2 Number 2 (53-57).

[11] Margaret H. Dunham "Data Mining Introductory and Advanced Topics".

[12] Corbett, A., \& Anderson, J. (1994). Knowledge Tracing: Modeling the Acquisition of Procedural Knowledge. User Modeling and User-Adapted Interaction, 4, 253-278. http://dx.doi.org/10.1007/BF01099821.

[13] Baker, R. and Aleven, V. 2008. Improving contextual models of guessing and slipping with a truncated training set. In Educational Data Mining 2008. 1st International Conference on Educational Data Mining, Proceedings. UNCCharlotte, Computer Science Dept., Montreal, Canada, 67-76.

[14] Baker, R. Corbett, A.T Keodinger, K., \& Wagner, A.Z. Off-task behavior in the cognitive tutor classroom: When students game the system. In: Proceeding of the SIGCHI Conference In human Factors in Computing Systems. 2004. (pp. 383-390).

[15] O.G. Selfridge. Pandemonium: a paradigm for learning. In Proceedings of the Symposium on Mechanisation of Thought Processes (pp. 511-529). Her Majesty's Stationery Office, London. 1959.

[16] M. Minsky. The society theory of thinking. In Artificial intelligence: an MIT perspective (pp. 423-450). MIT Press. 1979.

[17] Wooldridge, M. and Jennings, N.R. (1995). Intelligent Agents: Theory and Practice. Knowledge Engineering Review, 10(2), 115-152.

[18] Leap Motion Controller. Available online: https://www.leapmotion.com (accessed on 21 October 2014).

[19] JAVA Agent Development Framework. Available on line: http://jade.tilab.com/ (accessed on 21 October 2014).

[20] Drools a Business Rules Management System. Available on line: http://www.drools.org/ (accessed on 21 October 2014).

[21] Weka : Data Mining Software in Java Available on line http://www.cs.waikato.ac.nz/ml/weka// (accessed on 21 October 2014).

[22] Ángel S, Javier T, Pablo M, Baltasar F (2014): Application of Learning Analytics in Educational Videogames. Entertainment Computing, Elsevier. Volume 5, Issue 4, December 2014, Pages 313-322.

[23] A,Serrano-Laguna, A.B, Fernández-Manjón. (2014). Applying learning analytics to simplify serious games deployment in the classroom. Proceedings of the 2014 IEEE Global Engineering Education Conference (EDUCON) Pages 872-877. 


\section{BIOGRAPHIES OF AUTHORS}

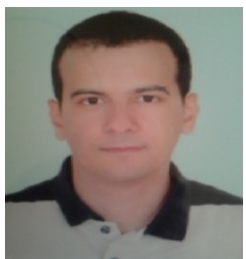

Lotfi Elaachak Received the "Master of Sciences and Technologies" degree in computer science engineering from the University Adbelmalek Essaadi of Tangier in 2009. He is currently working toward the Ph.D. degree with the Computing and Telecommunications Research Lab at Abdelmalek Essaadi University. His research interests include serious games, artificial intelligence and e-learning.

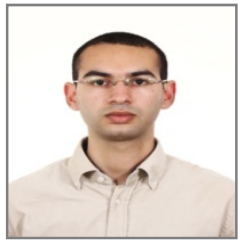

Amine Belahbib Received the "Master of Sciences and Technologies" degree in computer science Engineering from the University Adbelmalek Essaadi of Tangier, Morocco in 2012, and "Master of computer sciences" from Ceri of the University of Avignon, France in 2012. He is currently working toward the Ph.D. degree with the Computing and Telecommunications Research Lab at Abdelmalek Essaadi University. His research interests include serious games, artificial intelligence and e-learning.

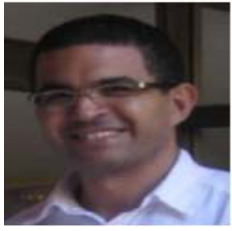

Mouhammed Bouhorma Prof. Dr. received the "Master degree in Electronics" degree in 1990 from Abdelmalek Essaadi University in Tetuan, Morocco, the "DEA" degree in Electronics and Telecommunications and the "Doctorat d'Etat" degree in Telecommunications with honors, respectively, in 1991 and 1995 from the "ENSEEIHT INPT" of Toulouse. He is a Professor of computer sciences and networks at Abdelmalek Essaadi University since 1999. Bouhorma has supervised several $\mathrm{PhD}$ and Masters Theses and has been the principal investigator and the project manager for several international research projects dealing with different research topics. 